\title{
ZINC-DEPRIVATION, MOTOR ACTIVITY AND ATTENTION
}

The effects of moderate dietary zinc deprivation (2 $\mathrm{mcg} / \mathrm{gm} \mathrm{diet})$ compared to an adequate zinc intake $(50 \mathrm{mcg} / \mathrm{gm})$ were compared using 24hour activity patterns and an attention task performance in 10 adolescent female monkeys (18-33 months of age) at the California Regional Primate Research Center, University of California, Davis, CA. Zinc deprivation caused a progressive decrease in daytime activity levels and impaired attention followed by growth retardation. (Golub MS, Takeuchi PT, Keen CL, Hendricks AG, Gershwin ME. Activity and attention in zinc-deprived adolescent monkeys. Am I Clin Nutr Dec 1996;64:908-915). (Reprints: Dr Mari S Golub, California Regional Primate Research Center, University of California, Davis, CA 95616).

COMMENT. Motor activity levels and attention may be decreased during early stages of zinc deprivation and before the onset of growth retardation. The authors cite references to estimates of zinc deficiency in $50 \%$ of US children, and especially in adolescent girls, $80 \%$ of whom consume less than the recommended dietary allowance of $12 \mathrm{mg} /$ day. Behavioral effects of zinc malnutrition may be observed before the more obvious onset of growth retardation. Children who eat primarily cereal proteins and little meat may be susceptible to zinc deficiency syndrome. (Millichap JG. Environmental Poisons in Our Food. Chicago, PNB;1993:62). ADD without hyperactivity is more prevalent than ADHD in girls compared to boys, and zinc deficiency should be considered as a factor in the etiology of inattentiveness, especially in girls.

\section{SEIZURE DISORDERS}

\section{PYRIDOXINE-DEPENDENT SEIZURES AND INTELLIGENCE}

Clinical manifestations, MRI abnormalities, learning disabilities, and effect of pyridoxine dose on intelligence quotients were studied in 6 definite and 3 possible cases of pyridoxine dependent seizures in children, ages 2.5 to 14 years, seen at Newcastle General Hospital, Newcastle upon Tyne, UK. Additional presenting features included jitteriness, encephalopathy, neonatal dystonia, hepatomegaly, and abdominal distension with bilious vomiting. Abnormal fetal movements, a hammering sensation, were noted in 4 . Later complications included break-through seizures with fever, visual agnosia, squint, articulatory apraxia, motor delay and dyspraxia, macrocephaly, and hydrocephalus. MRI showed focal thinning of the posterior third of the corpus callosum, cerebellar hypoplasia, and mild cerebral atrophy. Psychometric tests revealed specific impairments of expressive language with relative preservation of receptive verbal comprehension. IQ scores were below average (Full scale 50 to 73 ), but showed improvements after pyridoxine dose increases, particularly in the performance subscale. EEG abnormalities disappeared following pyridoxine therapy. (Baxter P, Griffiths P, Kelly T, Gardner-Medwin D. Pyridoxine-dependent seizures: demographic, clinical, MRI and psychometric features, and effect of dose on intelligence quotient. Dev Med Child Neurol Nov 1996;38:998-1006). (Respond: Dr P Baxter, Department of Paediatrics, Northern General Hospital, Herries Road, Sheffield S5 7AU, UK).

COMMENT. The diagnosis of pyridoxine-dependent seizures is based on clinical features and an absolute response to pyridoxine, recurrence after pyridoxine withdrawal, and immediate control after re-introduction. Language and cognitive disabilities may be partially reversible with optimal dosage. 